\title{
Gray-level co-occurrence matrix analysis of several cell types in mouse brain using resolution-enhanced photothermal microscopy
}

Takayoshi Kobayashi

Durga Sundaram

Kazuaki Nakata

Hiromichi Tsurui 


\title{
Gray-level co-occurrence matrix analysis of several cell types in mouse brain using resolution-enhanced photothermal microscopy
}

\author{
Takayoshi Kobayashi, ${ }^{a, b, c, d, \star}$ Durga Sundaram, ${ }^{a, e}$ Kazuaki Nakata, ${ }^{a}$ and Hiromichi Tsurui ${ }^{f}$ \\ aUniversity of Electro-Communications, Advanced Ultrafast Laser Research Center, Chofu, Tokyo, Japan \\ bJapan Science and Technology Agency, Core Research for Evolutional Science and Technology, K's Gobancho, Chiyoda-ku, Tokyo, Japan \\ 'National Chiao-Tung University, Department of Electrophysics, Hsinchu, Taiwan \\ dOsaka University, Institute of Laser Engineering, Suita, Osaka, Japan \\ eSastra University, Tirumalaisamudram, Thanjavur, Tamil Nadu, India \\ fJuntendo University School of Medicine, Department of Pathoilogy, Bunkyo-ku, Tokyo, Japan
}

\begin{abstract}
Qualifications of intracellular structure were performed for the first time using the gray-level co-occurrence matrix (GLCM) method for images of cells obtained by resolution-enhanced photothermal imaging. The GLCM method has been used to extract five parameters of texture features for five different types of cells in mouse brain; pyramidal neurons and glial cells in the basal nucleus (BGI), dentate gyrus granule cells, cerebellar Purkinje cells, and cerebellar granule cells. The parameters are correlation, contrast, angular second moment (ASM), inverse difference moment (IDM), and entropy for the images of cells of interest in a mouse brain. The parameters vary depending on the pixel distance taken in the analysis method. Based on the obtained results, we identified that the most suitable GLCM parameter is IDM for pyramidal neurons and BGI, granule cells in the dentate gyrus, Purkinje cells and granule cells in the cerebellum. It was also found that the ASM is the most appropriate for neurons in the basal nucleus. $\odot$ The Authors. Published by SPIE under a Creative Commons Attribution 3.0 Unported License. Distribution or reproduction of this work in whole or in part requires full attribution of the original publication, including its DOI. [DOI: 10.1117/1. JBO.22.3.036011]
\end{abstract}

Keywords: microscopy; superresolution; neurons; cells; cell analysis; image analysis.

Paper 170031R received Jan. 20, 2017; accepted for publication Feb. 17, 2017; published online Mar. 14, 2017.

\section{Introduction}

Optical microscopy has been extensively used for biological imaging for many years but the resolution is limited by diffraction. ${ }^{1}$ Recently, in order to visualize a variety of nanoscale cellular components with high specificity and high spatial resolution, several superresolution imaging techniques such as stimulated emission depletion microscopy, structured illumination microscopy, and stochastic optical reconstruction microscopy have been developed reaching sub-100-nm resolution. ${ }^{2-7}$ Such high resolution has revealed fine intracellular structures. Another direction of the development of microscope is the imaging of nonfluorescent system with high sensitivity and resolution using photothermal (PT) effects with resolution enhancement. ${ }^{8-12}$ In these studies, although the high resolution capability thanks to the pump-probe scheme has revealed fine intracellular structures, intracellular "global" statistical features of the intracellular structure such as uniformity, correlation among the microstructures, and entropic randomness have not been investigated. For example, even though analysis has been performed with the fractal dimensions. ${ }^{13}$ In this case, however, superresolution was not appreciably utilized and the analysis was only applied to two-dimensional (2-D) images. ${ }^{13}$ The accurate detection of a deformation or structural changes at the cellular level depends on the efficiency of the microscope and the skill of the

*Address all correspondence to: Takayoshi Kobayashi, E-mail: kobayashi@ils uec.ac.jp investigator and is prone to human error. A quantitative measure for the classification and detection of some cell types from the pathological viewpoint at the cellular level has been established using a microscope and texture analysis using the gray-level co-occurrence matrix (GLCM) method. The GLCM method is capable of evaluating fine structural changes in the organelles in cells or in the distribution of cells that are otherwise difficult to properly evaluate using standard visual analysis methods. Thus far, GLCM analysis has been performed using differences in the texture parameters of images obtained through standard microscopic methods. For example, the GLMC method has been used to study the distribution arrangements of cells in two areas of the mouse brain, the cortex and the medulla. ${ }^{14,15}$ However, GLCM-type analysis has not been utilized to study high resolution intracellular images.

In this study, we performed for the first time qualifications of intracellular structure in the resolution-enhanced images of cells in mouse brain obtained by PT imaging, which we developed to perform nondiffraction limited imaging using a compact system without requiring fluorescent capability. ${ }^{16-18}$ We examined five types of cells, neurons, and glia cells in basal nuclei, Purkinje cells and granule cells in cerebellum, and granule cells in dentate gyrus. Those cells are distinct in their morphology and the location, which provides reliable identification of them. Those cells have been shown to be involved in peculiar diseases: Purkinje cells in Niemann-Pick type $\mathrm{C} 1$ ataxia and spinocerebellar degeneration type 6 , cerebellar granule (CGr) cells in ataxia telangiectasia, both neurons and glia cells of basal nuclei 


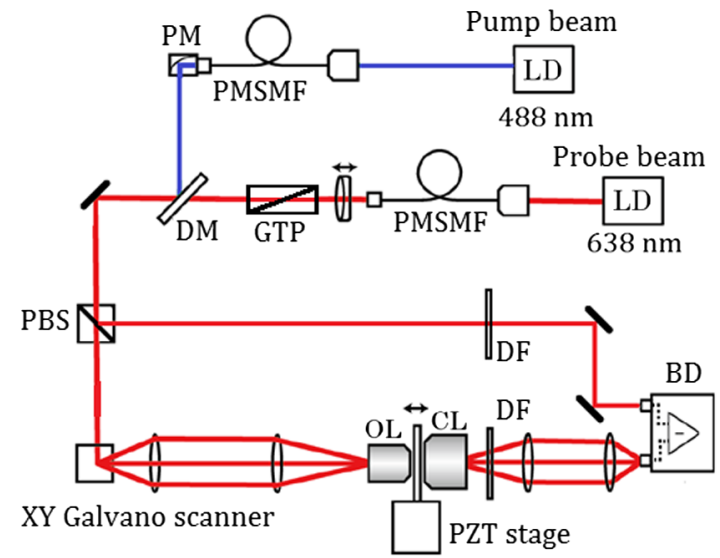

Fig. 1 Schematic of the system of a PT microscope equipped with a Galvano scanner and spatially segmented balanced detection. LD, laser diode; PMSMF, polarization maintaining single-mode fiber; PM, parabolic mirror; GTP, Glan-Thompson polarizer; DM, dichroic mirror; PBS, polarizing beam splitter; OL, objective lens; CL, condenser lens; DF, dielectric multilayer filter; and BD, balanced detector.

in corticobasal degeneration, dentate gyrus granule (DGr) cells in Alzheimer disease.

There have been some reports which correlate GLCM analysis parameters to physiological or pathological meanings: finite nuclear change associated with thymocyte development, ${ }^{14}$ correlation with cancer prognosis ${ }^{19}$ or grading, ${ }^{20}$ and nuclear change in the early stage of apoptosis. ${ }^{21}$ However, those findings are correlations between GLCM parameters and are rather superficial phenomena, and underlying mechanisms are not shown. This is due to the stage at which GLCM analysis has been accomplished and where it still has substantial pathological or medical meanings. In this paper, we have successfully assigned parameters which are appropriate to characterize individual cells.

As the texture properties obtained by the GLCM method vary depending on distance, parameter $d$ was selected for analysis. ${ }^{14,15}$ Our results exhibit a characteristic variation of the dependence of the five GLCM parameters on $d$. For analysis, we evaluated the parameters for the five images taken in the order of increasing depth from layer 1 to layer 5 in steps of $0.283 \mu \mathrm{m}$ in the same lateral region of interest.

This study has been reviewed and approved with License number 280076 by the Institutional Animal Care and Committee of Juntendo University. All the authors have no relevant financial interests in the manuscript and no other potential conflicts of interest to disclose.

\section{Experiment}

We performed pump-probe microscopy using an intensitymodulated laser diode (LD) as a pump and a nonmodulated continuous-wave LD as a probe. The schematic of the system is shown in Fig. 1 and the details are described below.

The principle of the pump-probe microscopy is based on the PT effect induced by the pump and detected by the probe. The absorbed pump beam energy with Gaussian distribution on the focal plane is converted to heat and changes the refractive index forming a concave thermal lens which deflects the probe beam resulting in the detected probe intensity change. High spatial resolution can be obtained for the pump-probe microscope because the signal intensity is proportional to the product of the pump and probe intensities. ${ }^{10}$ The sensitivity of the PT microscope is reported to be a single-molecular level even at room temperature. ${ }^{22}$

The details of the PT microscopy system, which uses simple LDs and a balance detector, are described in our previous papers. ${ }^{16,17}$ Two modes of imaging were used: stage scanning and fast Galvano scanning. These modes are described below.

In the stage-moving mode, each horizontally linear polarized beam was collimated through a spatial filter, and the two beams were combined with a dichroic beam-combining mirror. The sample position was raster-scanned using a three-axis positioning stage driven by piezoactuators (Thorlabs MAX311D). To evaluate the spatial resolution using gold nanoparticles (GNPs), we used a high-accuracy positioning stage (PI P-622.2) with a closed-loop resolution of $0.7 \mathrm{~nm}$. A 1.4-NA oil-immersion condenser (Olympus U-AAC) was used to collect the transmitted probe light. A narrowband filter matching the laser line was placed in front of the detector to filter out both the excitation beam and the fluorescence from the sample, so that only the probe beam was detected. An autobalanced photoreceiver (New Focus, Nirvana) was used to cancel out the noise due to intensity-fluctuation of the probe laser. The probe beam was reflected by a beam splitter placed in front of the focusing lens which reflected the probe beam and then directed it to the reference port of the balanced detector through a multimode fiber. The maximum area size available in the stage scan mode is $20 \mu \mathrm{m} \times 20 \mu \mathrm{m}$. Image sizes typically described in this paper are $144 \times 144$ points, which corresponds to $3.6 \mu \mathrm{m} \times 3.6 \mu \mathrm{m}$. The lock-in amplifier sensitivity was $5 \mathrm{mV}$, and the time constant was $500 \mu$ s per point.

In the beam-sweeping mode with the Galvano-mirror system, the collimated pump laser and the probe laser through the Glan-Thompson polarizer were overlapped through a dichroic mirror. The probe beam was split with a polarizing beam splitter (PBS) for balance detection and was reflected with the Galvano mirror. One of the split probe beams was used as a reference beam. The center of the 4 f-optical system was set at the sample position. The probe transmitting through and reference bypassing the sample were both detected with the split detection section of the balance detector. Three-dimensional (3-D) images of the samples were obtained by scanning in the $X-Y$ plane with the Galvano mirror and by moving the piezo-stage in the $Z$-direction. The probe beam through the sample and the reference beam irradiated the balance detector. Image sizes in this system are $300 \times 300$ pixels corresponding to $72 \mu \mathrm{m} \times 72 \mu \mathrm{m}$. In comparison with the $3.6 \mu \mathrm{m} \times 3.6 \mu \mathrm{m}$ of the stage-scan mode, the Galvano system can cover a much wider range. The lock-in amplifier sensitivity was $5 \mathrm{mV}$, and the time constant was $20 \mu$ s per pixels. D-well time was $50 \mu$ s per pixel.

In both modes, two LDs [Nichia NDS4216 (488 nm) and Mitsubishi ML520G55 (638 nm)] were used for the pump and probe, respectively. Their direct output powers from the lasers were $\sim 200 \mathrm{~mW}$, which was reduced to $2.0 \mathrm{~mW}$ for the pump and $2.6 \mathrm{~mW}$ for probe at the focal point of the microscope by appropriate filters. The LDs were mounted on temperature-controlled laser-diode mounts (Thorlabs, LDM9T). An objective lens [Olympus, UPLSAPO 40X2, numerical aperture $(\mathrm{NA})=0.95]$ was used. The beam size was adjusted to fill the back aperture of the objective lens. The lock-in technique was utilized to detect the pump-induced signal modulation with a lock-in amplifier (Signal Recovery 7270) referenced to a frequency of $100 \mathrm{kHz}$. A modulated signal at the frequency 
of $100 \mathrm{kHz}$ was generated by the bilinear interaction between the pump field and the probe field in the sample. The output of the balanced detector was connected to the lock-in amplifier through a low-pass filter (Mini-circuit LPF-B0R3). The reference signal for the lock-in amplifier was delivered from the signal generator.

GNPs of 20-nm diameter were used for the evaluation of resolution. GNPs were dispersed in water and mixed with an aqueous solution of polyvinyl alcohol. Then the mixed solution is spin coated on a glass substrate.

The details of the tissue sample are described as follows. In this study, sagittal-sliced sections of mouse brain were used. When the sliced section was stained using the hematoxylineosin method, the cerebral cortex, the hippocampal formation under the corpus callous, basal nuclei, and the cerebellar portion can be easily identified by an individual well experienced in the anatomy of the mouse brain from their structures and the well-established analytical atlas of the mouse brain. Cells that were large and stained faintly in the basal nucleus (BN) were considered to be nerve cells (pyramidal cells). Cells in the $\mathrm{BN}$ that were small and stained heavily were considered to be glial cells. The hippocampal formation has a very distinctive shape, and cells included within the hippocampus can be easily identified. We captured images of cells, which were round and not bipolar or pyramidal, and which could be identified as granule cells in the gyrus dentate. In the cerebellar cortex, the border between the Purkinje cell layer and granular cell layer is clear. The granular cells are small and strongly stained, whereas Purkinje cells have large round bodies and thick processes. As such, they were easily identified. The purpose of this study is to demonstrate the capability of the proposed method for extracting the quantitative characteristic features of these cell types in different domains of a brain. This can be useful for the estimation of the degree of pathological process in various cells.

The GLCM analysis was performed using the image data obtained by the Galvano-mode imaging. We evaluated five images taken at five depths increasing from layer 1 to layer 5 with a step of $0.283 \mu \mathrm{m}$ while maintaining the same lateral region.

\section{Imaging Results}

First, we studied the spatial resolution of the PT microscope used in the present experiment by imaging GNPs of $20 \mathrm{~nm}$ in diameter as shown in Fig. 2. This image was taken in the moving stage mode. Figure 2(a), one prominent image, three medium intensity images, and two faint images are visible by stage moving measurement. The differences in intensity are due to the difference in the distance in the $Z$-direction from the central focal plane for these GNPs in the doped samples. Figures 2(b) and 2(c) show 2-D GNP images in the lateral plane and the axial direction by the Galvano-mirror system, respectively. The most intense GNP image was used for the resolution evaluation. The full-width at half-maxima (FWHMs) of the intensity distribution profile was found to be $219 \pm 9 \mathrm{~nm}$ for the piezo-stage system as shown in Fig. 2(d). The experimental resolution is defined as the FWHM of Gaussian function obtained by the least squares mean (LSM) fitting to the observed intensity distribution of the GNPs, and the values following " \pm " symbol are the standard deviations obtained by the LSM. If the finite size of the GNP $(20 \mathrm{~nm})$ is taken into account, the FWHM is $218 \pm 9 \mathrm{~nm}$.
A theoretical evaluation of the resolution of the PT microscopy was conducted for the purpose of comparison with the experimental results.

The "lateral" distribution of the signal intensity depends on the probe and heating beam profiles and also on the dielectric susceptibility profile of the target structured material (in the present case intracellular structure). However, the "lateral" spatial extension of the susceptibility profile is much smaller than the "lateral" size of the probe beam, and the transverse resolution detected using the phase sensitive detection method as in this study is concluded to be approximated by the product of the two (pump and probe) beam profiles. ${ }^{10}$ This procedure was applicable under the experimental conditions in the previous studies ${ }^{17,23,24}$ as well as in this study. The FWHMs of the intensity distribution of the pump $(488 \mathrm{~nm})$ and probe $(638 \mathrm{~nm})$ are calculated to be 264 and $350 \mathrm{~nm}$, respectively, using the Bessel function as the point spread function (PSF) with an NA $=0.95$ [see Fig. 2(g)]. Then, the FWHM of the pump-probe setup obtained by the product of these two Bessel functions corresponding to the pump and probe was calculated to be $210 \mathrm{~nm}$. The resolution obtained by the PT microscopy of the GNP in this study was determined to be $219 \pm 9 \mathrm{~nm}$ for the piezo-stage system in relatively good agreement with the calculation result of $210 \mathrm{~nm}$. Thus, it is proved that the intensity distribution of the PT microscopy image is approximately given by the product of the PSFs of pump and probe. This means that the resolution is not limited by the spot size of the pump beam $(264 \mathrm{~nm})$ but is rather limited by the product of the PSFs of the pump and probe. This indicates that the imaging is beyond the diffraction of both pump and probe beams. Higher resolution is realized by nonlinear PT imaging of the combination of the present resolution enhancement mechanism and nonlinear effect. ${ }^{18}$ This resolution improvement is also found in other pump-probe microscopies such as stimulated gain microscopy and stimulated emissioninduced fluorescence-quenching microscopy. ${ }^{16}$

The FWHM of the intensity distribution profile was found to be $270 \pm 8 \mathrm{~nm}$ for the Galvano-mirror system as shown in Fig. 2(e). When including the effect of the GNP size, FWHM of the intensity distribution profile was $269 \pm 8 \mathrm{~nm}$. The degradation of resolution in the Galvano system $(269 \mathrm{~nm})$ from the piezo-stage stage system $(209 \mathrm{~nm})$ likely occurs because the inertia of a galvanometric scanner leads to time delays when following external commands, which subsequently introduces image distortions that deteriorate as the scan frequency increases. $^{25}$

Thus, the lateral resolution is well explained in terms of the product of the PSFs of pump and probe. On the other hand, the axial resolution is not given by this product. ${ }^{10}$ It was found both theoretically and experimentally that the assumption of a product PSF of heating and detection laser in the axial direction is inappropriate for describing the PT image profile. Instead, the two-lobe structure and the size of the two lobes depend sensitively on the axial displacement $\left(\Delta z_{f}\right)$ of the two laser foci [Figs. 2(d) and 2(e) in Ref. 10]. It was found that independently of $\Delta z_{f}$, whenever the probe laser focus is in front of the refractive index profile, the detected intensity is decreased as compared to that of the undisturbed probe beam, while a signal increased intensity is measured if the probe laser focus is behind the refractive index profile [Fig. 1(c) in Ref. 10]. The two lobes are shown in Figs. 2(c) and 2(f). There is some peak displacement (in the $X$-direction) along the $Z$-direction in Fig. 2(c). 
(a)

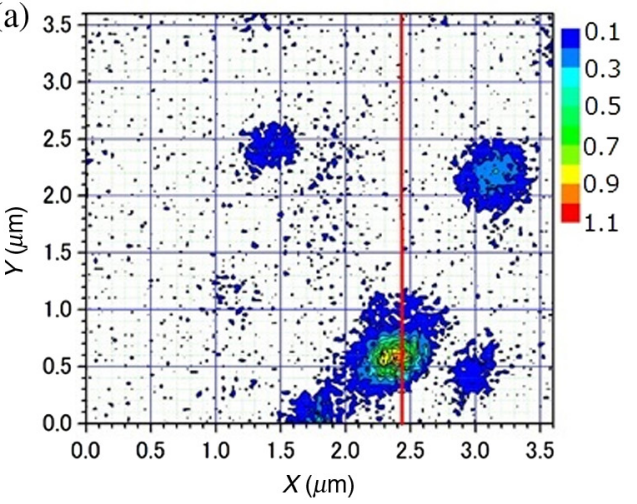

(d)

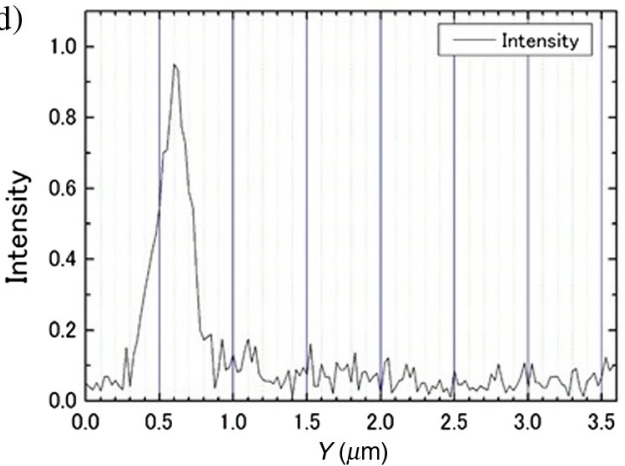

(f)

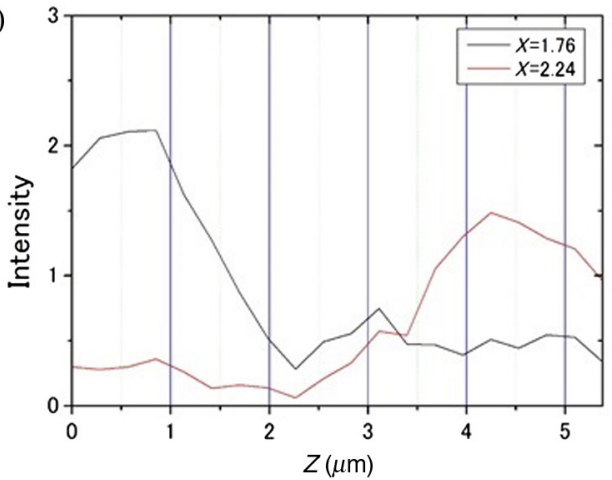

(b)
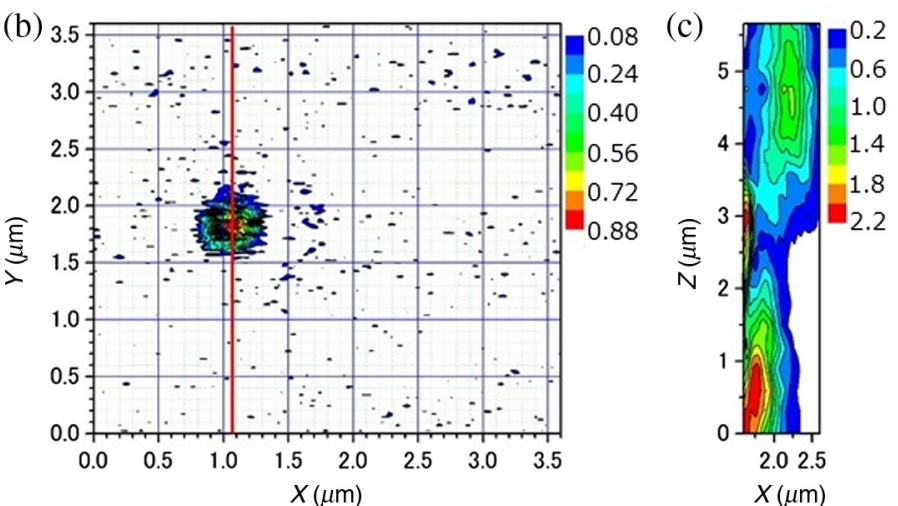

(e)

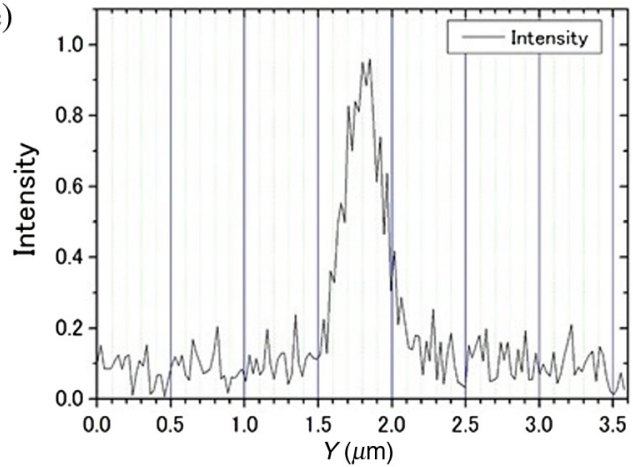

$(\mathrm{g})$

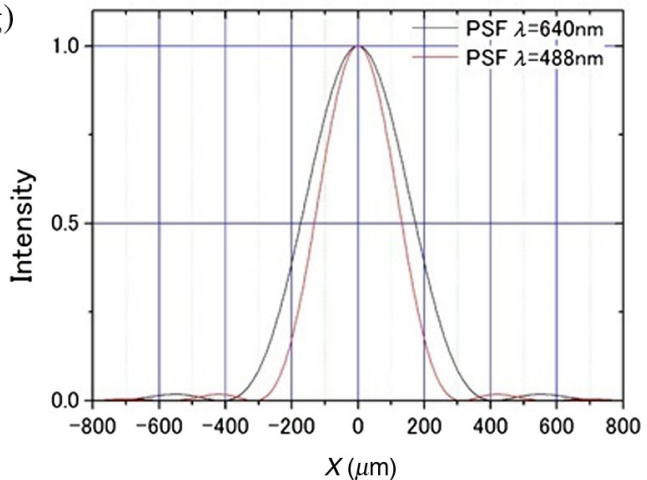

Fig. 2 (a) 2-D GNP image obtained by PT microscopy in the piezo-stage moving mode (a) and (d) intensity distribution profile along the red line in (a); (b) 2-D display of a GNP image in the $X Y$-plane obtained by PT microscopy in the Galvano-sweeping mode, (b) intensity distribution profile along the red line in (b); (c) 2-D display of a GNP image in $X Z$-plane measured with PT microscopy in the Galvano-sweeping mode; (e) intensity distribution profile at $x=1.76$ and $x=2.24$ in (f); (g) calculated PSF at $\lambda=638$ and $488 \mathrm{~nm}$.

This is probably due to some small dislocation of the beam axis between pump and probe in the $X$-direction.

\section{Texture Analysis}

The analysis of textures of the five different cell types described below was performed by using the GLCM method. ${ }^{14,15}$ The texture parameters were calculated by analyzing relevant regions of interest of five 2-D images of the mouse brain of interest captured at increasing depths from layer 1 to layer 5 with a step of $0.283 \mu \mathrm{m}$ in the axial direction. The PT images of neurons in BN [Figs. 3(a)-3(i)] and cerebellum [Figs. 4(a)-4(i)] were captured by step-wise shifting along the axial direction with a step of $283 \mathrm{~nm}$. Images (j) in both Figs. 3 and 4 show brightfield images of the corresponding region to the PT images.
Figure 4(k) shows a bright-field image of the cerebellum in a different place from (a) to (j). Purkinje cells are lined side by side next to the granule cell layer. Figure 5(a)-5(i) and 5(j) show the PT images and bright-field image, respectively, of the hippocampal dentate gyrus. Image (k) in Fig. 5 shows the PT image of the dentate gyrus in an extended area, which is the same area as in (i) showing the overplayed bright-field image and red-boarded area image in (j) in Fig. 5.

We studied five different cell types; a neuron (nerve cell), a glial cell, two kinds of granule cells, and a Purkinje cells. The fundamental properties and functions of them are briefly described as follows. The numbers of five cell types studied were 10 for pyramidal neurons in the BN,10 for glial cells in the basal nucleus (BGl), 5 for cerebellar Purkinje (CP) cells, 8 for CGr cells, and 32 for DGr cells. 

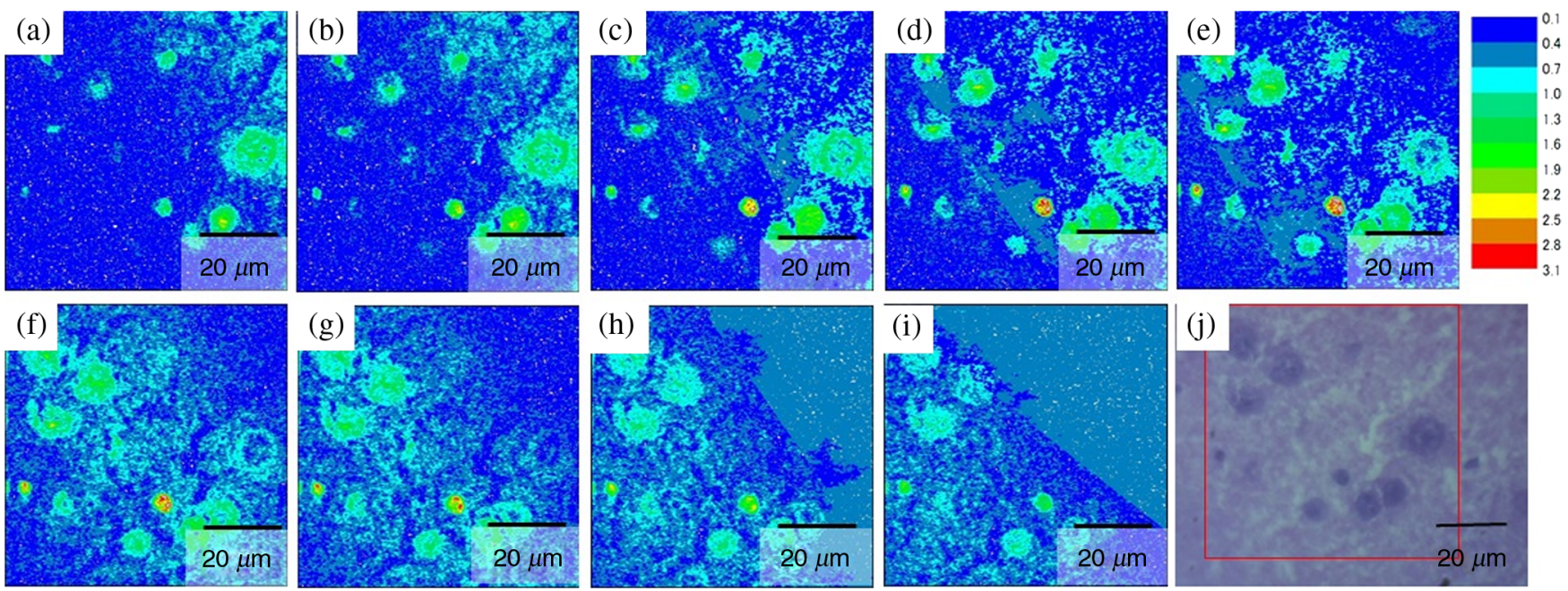

Fig. 3 PT images and bright-field image of neurons in BN. Nine images (a)-(i) are taken by shifting along the axial direction by $283 \mathrm{~nm}$ at each step. (j) The bright-field image. Red-line marked box in image (j) showing the image area of (a)-(i).
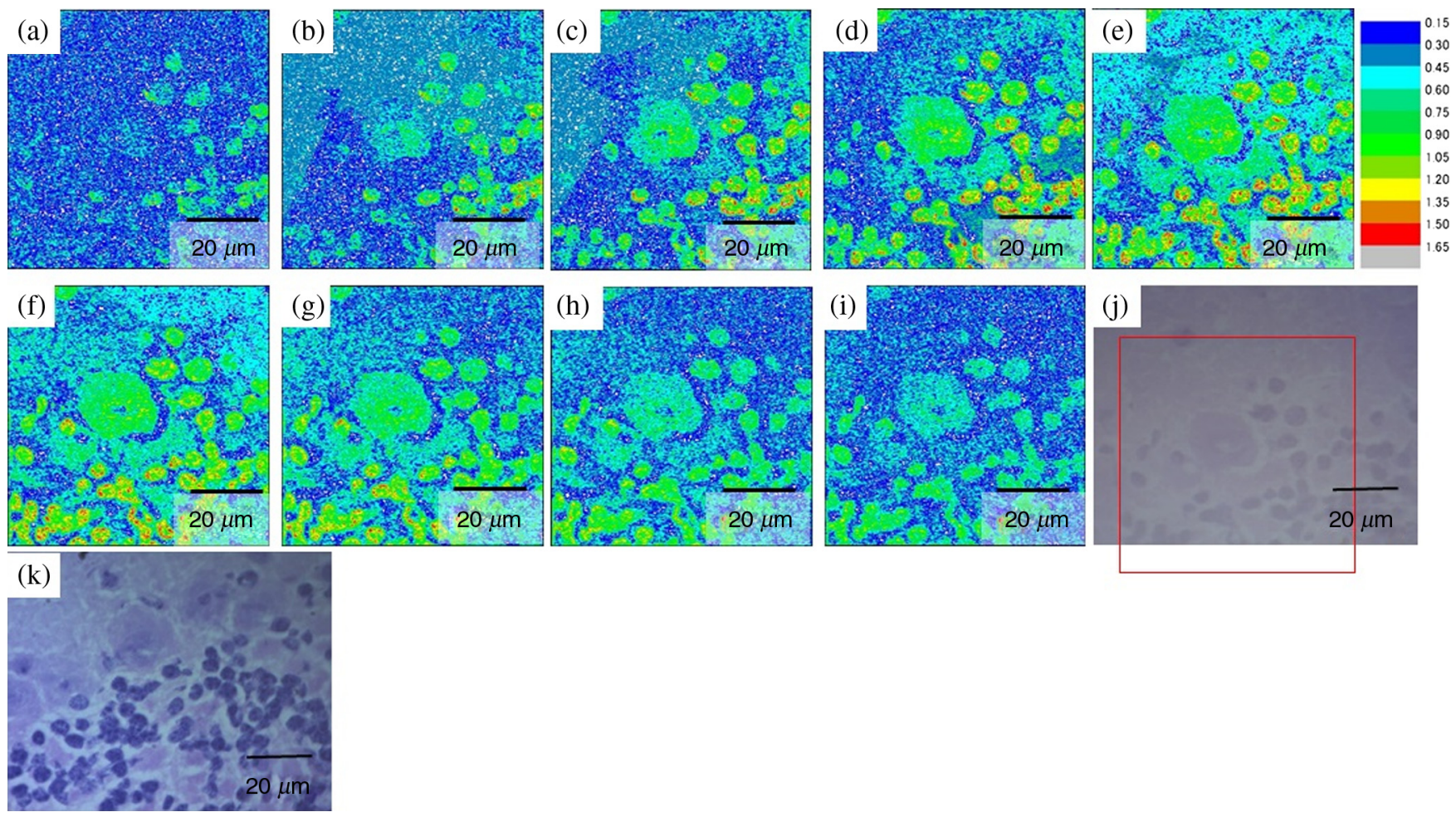

Fig. 4 PT images and bright-field image of neurons in cerebellum. Nine images (a)-(i) are taken by shifting along the axial direction by $283 \mathrm{~nm}$ at each step. (j) The bright-field image. Red-line marked box in image (j) showing the image area of (a)-(i). (k) A bright-field image of the cerebellum in a different place from (a)-(j). Purkinje cells lined with layers and many granule cells are shown in (k).

In Fig. 6, analysis was performed for (a) nerve cells and (b) glial cells in the BN in cerebrum, (c) Purkinje cells and (d) granule cells in the cerebellum, and (e) granule cells in the dentate gyrus. The inside areas of the encircled parts were used for the analysis.

Figure 6 shows the images taken by the PT microscope for $\mathrm{BN}$ and $\mathrm{BGl}, \mathrm{CP}$ and CGr, and DGr. The black lines encircling single cells in the domain show the area where the analysis was made. The encircling of the cell was performed by thresholding setting for the digitized 2-D image data of 256 gray levels.

Here, we describe briefly the function of the studied cell types.

Nerve cells are specialized cells found in the nervous system designed to stimulate other cells in the body in order to communicate. Neurons are excitable, which means that they function by using electrical stimulation. Glial cells are 

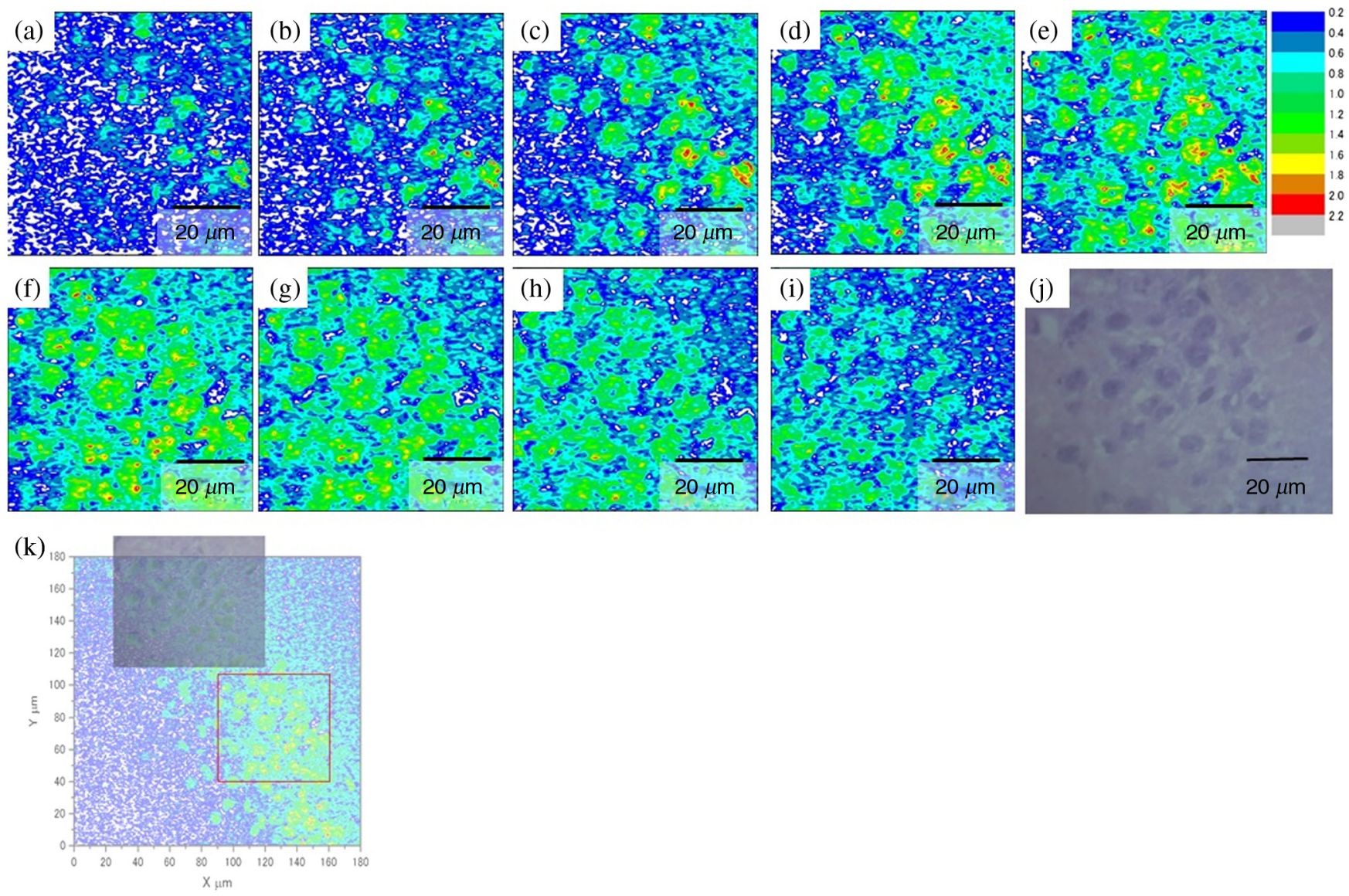

Fig. 5 PT image and bright-field image of dentate gyrus. Nine images (a)-(i) are taken by shifting along the axial direction by $283 \mathrm{~nm}$ at each step. (j) A bright-field image. (k) shows the extended PT image with an overlaid bright-field image and red-line marked box showing the image area of (a)-(i).

nonneuronal cells that maintain homeostasis, form myelin, and provide support and protection for neurons in the central and peripheral (called Schwan cells) nervous systems. Purkinje cells, also called Purkinje neurons, are neurons in vertebrate animals located in the cerebellar cortex of the brain. Purkinje cell bodies are shaped like a flask and have many threadlike extensions called dendrites, which receive impulses from other neurons called cerebellar granule cells. Granule cells of the cerebellum are among the smallest neurons in the brain. (The term granule cell is used for several unrelated types of small neurons in various parts of the brain.) CGr cells are the most numerous neurons in the brain. Granule cells in the molecular layer of the dentate gyrus receive the hippocampal formation's major excitatory input from the cortex. To compare the difference in intracellular structures among the five cell types, we analyzed the PT images by the GLCM method.

The GLCM is a square matrix in which the axes correspond to the number of gray levels in an image. It contains the frequency of neighboring pixel relationships of a 2-D image (of the region of interest) at the position of the corresponding gray level. The value of frequency at which the pixel intensity pair $i$ and $j$ appear together is denoted by $P(i, j)$ (see the detailed definition of this value in Refs. 14 and 15). Several texture properties can be extracted from the GLCM matrix, including angular second moment (ASM), contrast, correlation, inverse difference moment (IDM), and entropy.
ASM is a measure of the homogeneity of an image

$$
\mathrm{ASM}=\sum_{i=0}^{G-1} \sum_{j=0}^{G-1}\{P(i, j)\}^{2}
$$

"ASM" value shows the strength of homogeneity, namely the pair correlation $[P(i, j)]$ of the whole area nearly independent of the distance between $i$ and $j$. So even if the concentration of chromatin in this case is homogeneous, it becomes smaller if the concentration has slope(s) in any direction showing area-wide uniformity. This can happen in the case of glial cells because the nuclei of glial cell are small and condensed resulting in the homogeneous distribution of chromatin. The average value of ASM measured for pixel distance $d=1$ was $0.272 \pm 0.087$ and was the highest for BGl. The values decreased to $0.229 \pm 0.085$ and $0.201 \pm 0.083$ when the pixel distance was increased to 2 and 3 , respectively. The difference in ASM between the cells was highest for BGl and CP, followed by $\mathrm{CGr}$ and $\mathrm{BN}$. CGr and $\mathrm{CP}$ had the least difference in magnitude of ASM for pixel lengths (distances) $d=1$ and 3, with a mean difference of 0.04. As can be expected from the images in Fig. 6, Purkinje cells have large and patterned nuclei, and the abovementioned special properties of its pattern are quantitatively discussed and quite reasonably explained. A homogeneous area is considered to contain only a small number of gray levels, 
(a)

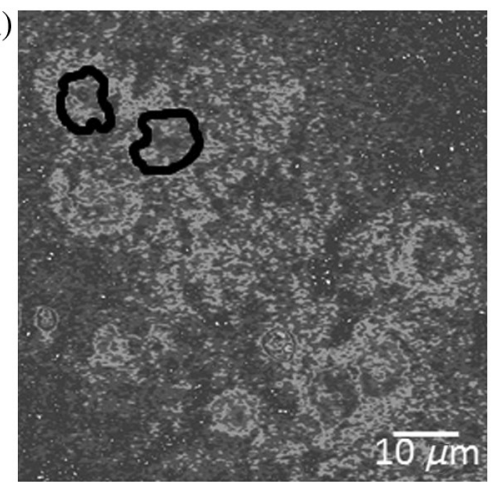

(c)

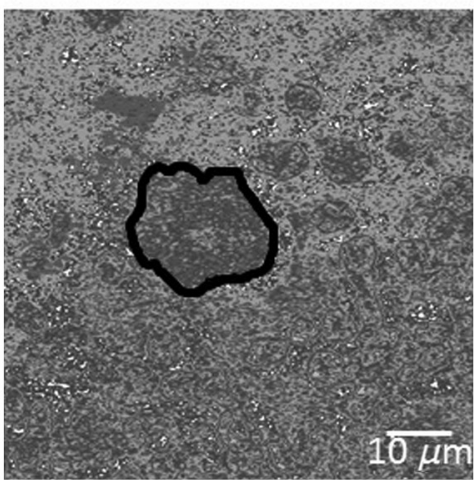

(b)

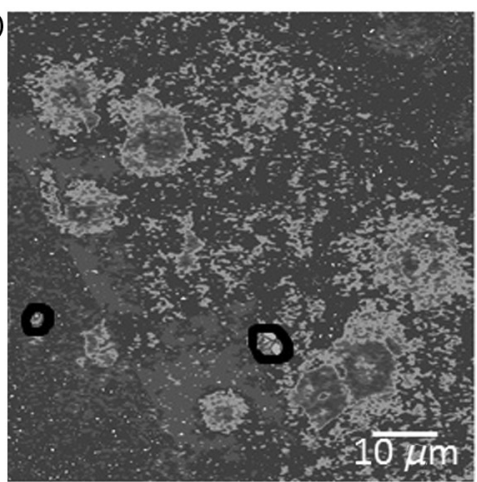

(d)

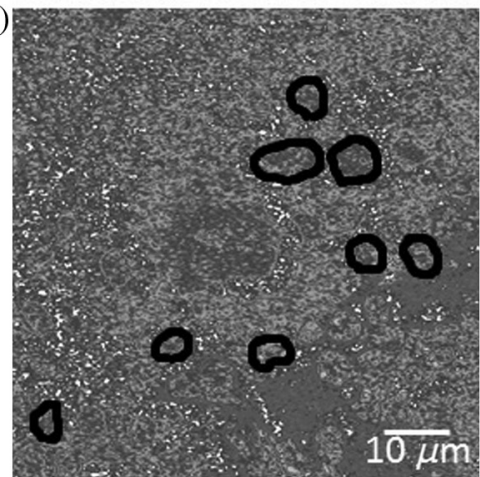

(e)

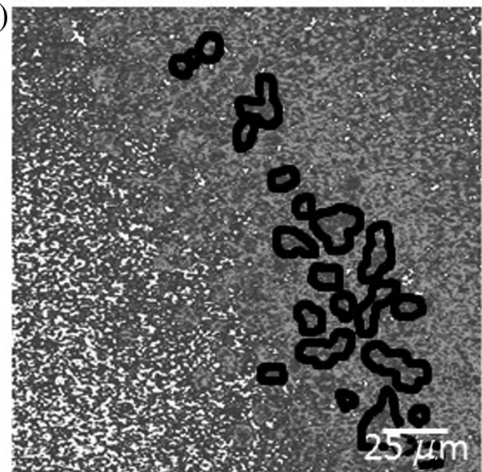

Fig. 6 Images taken with Galvano-mode PT microscopy in the domains of (a) and (b) cerebrum, (c) and (d) cerebellum, and (e) hippocampal dentate gyrus. The types of cells indicated by the encircling black lines are (a) nerve cells and (b) glial cells in the BN, (c) Purkinje cells, (d) granule cells in the cerebellum, and (e) granule cells in the dentate gyrus. The inside areas of encircled parts were used for the analysis.

giving a GLCM with only a few but relatively high values of $P(i, j){ }^{26-29}$ Thus, the sum of squares giving the values of ASM will be high.

Contrast is a measure of the local variations in gray levels present in an image. Images with large neighboring gray level differences are associated with a high contrast

contrast $=\sum_{|i-j|=0}^{G-1}|i-j|^{2}\left\{\sum_{i=1}^{G} \sum_{j=1}^{G} P(i, j)\right\}$.

Contrast by definition represents the difference in the concentration in the distal location due to the $|i-j|^{2}$ term. This means that if some cell has a large value of this parameter, within the cell of this type the concentration is relatively localized in some specific spatial area in the cell. Contrast of CGr is the highest among the five cell types in the case of $d=1-6$. It is to be noticed that the value is dependent on the distance parameter $d$ as in the other parameter. For example, the contrast of $\mathrm{BGl}$ is larger than the one of $\mathrm{BN}$ in the case of $d=1$, e.g., (396; BGl, $d=1)>$ contrast (383; BN, $d=1$ ). However, contrast $(915 ; \mathrm{BGl}, d=3)<$ contrast $(976 ; \mathrm{BN}, d=3)$ (Table 1). The crossover takes place between $d=1$ and $d=2$. This shows that the evaluation of difference of $P$ varies with $d$. This dependence also provides the important information of the dependence of difference distribution with the unit of $d$ (distance parameter for GLCM analysis $=283 \mathrm{~nm}$ ) in the GLMC analysis, which is very reasonably used since it is close to the PT microscope resolution $(219 \mathrm{~nm})$.

Correlation is a measure of gray level linear dependence between pixels at specified positions relative to each other correlation $=\sum_{i=0}^{G-1} \sum_{j=0}^{G-1}\left[\frac{\{i \times j\} \times P(i, j)-\left\{\mu_{x} \times \mu_{y}\right\}}{\sigma_{x} \times \sigma_{y}}\right]$.

Here, $\mu_{x}$ and $\mu_{y}$ are, respectively, the means of $P_{x}(i)=$ $\sum_{j=0}^{G-1} P(i, j)$ and $P_{y}(j)=\sum_{i=0}^{G-1} P(i, j)$, and $\sigma_{x}$ and $\sigma_{y}$ are the corresponding standard deviations.

Correlation is different from contrast and IDM in such a way that it is the difference from the mean values of $P(i, j)$, while the other two are the absolute values. Therefore, in case the frequency $P(i, j)$ is increased or decreased with the same amount for all of the $P(i, j)$ pairs, the values of contrast and IDM proportionally increase or decrease correspondingly, while correlation is maintained.

The correlation of $\mathrm{BGl}$ at pixel distance 1 is $(89.9 \pm 3.1) \times 10^{-5}$, which was the highest among all five cell types studied. The value decreased to $(70.3 \pm 5.9) \times 10^{-5}$ when the pixel distance was increased to 2 . DGr had an average correlation value of $(88.8 \pm 4.6) \times 10^{-5}$ for distance 1 and $(85.9 \pm 5.0) \times 10^{-5}$ for distance 2 . The difference in the average correlation value between CGr and BGl was the highest in the case of $d=1$. The value of correlation decreased for all cells with an increase in pixel distance value.

The IDM defined by the following equation is also influenced by the homogeneity of the image

$\mathrm{IDM}=\sum_{i=0}^{G-1} \sum_{j=0}^{G-1} \frac{1}{1+(i-j)^{2}} P(i, j)$. 
Table 1 Values of texture parameters evaluated for three pixel distances $d=1,2,3$. The five cell types studied were pyramidal neurons in the BN, BGI, DGr cells, CP cells, and CGr cells.

\begin{tabular}{|c|c|c|c|c|}
\hline Cell type & Texture parameter & $d=1$ & $d=2$ & $d=3$ \\
\hline \multirow[t]{5}{*}{$\mathrm{BN}$} & ASM & $0.171 \pm 0.022$ & $0.136 \pm 0.021$ & $0.115 \pm 0.02$ \\
\hline & Contrast & $383 \pm 39$ & $720 \pm 69$ & $976 \pm 88$ \\
\hline & Correlation & $(77.8 \pm 6.9) \times 10^{-5}$ & $(62.8 \pm 5.6) \times 10^{-5}$ & $(51.4 \pm 4.6) \times 10^{-5}$ \\
\hline & IDM & $0.776 \pm 0.026$ & $0.668 \pm 0.038$ & $0.590 \pm 0.046$ \\
\hline & Entropy & $3.31 \pm 0.19$ & $3.37 \pm 0.20$ & $3.40 \pm 0.20$ \\
\hline \multirow[t]{5}{*}{ BGI } & ASM & $0.272 \pm 0.087$ & $0.229 \pm 0.085$ & $0.201 \pm 0.083$ \\
\hline & Contrast & $396 \pm 64$ & $703 \pm 81$ & $915 \pm 78$ \\
\hline & Correlation & $(89.9 \pm 3.1) \times 10^{-5}$ & $(70.3 \pm 5.9) \times 10^{-5}$ & $(53.7 \pm 6.5) \times 10^{-5}$ \\
\hline & IDM & $0.797 \pm 0.034$ & $0.699 \pm 0.051$ & $0.629 \pm 0.063$ \\
\hline & Entropy & $2.85 \pm 0.39$ & $2.90 \pm 0.40$ & $2.93 \pm 0.40$ \\
\hline \multirow[t]{5}{*}{$\mathrm{CP}$} & ASM & $0.088 \pm 0.005$ & $0.061 \pm 0.003$ & $0.048 \pm 0.003$ \\
\hline & Contrast & $631 \pm 96$ & $1131 \pm 160$ & $1461 \pm 188$ \\
\hline & Correlation & $(69.9 \pm 7.6) \times 10^{-5}$ & $(46.0 \pm 6.4) \times 10^{-5}$ & $(30.3 \pm 5.3) \times 10^{-5}$ \\
\hline & IDM & $0.595 \pm 0.005$ & $0.447 \pm 0.007$ & $0.360 \pm 0.008$ \\
\hline & Entropy & $4.40 \pm 0.08$ & $4.44 \pm 0.08$ & $4.47 \pm 0.08$ \\
\hline \multirow[t]{5}{*}{ CGr } & ASM & $0.091 \pm 0.009$ & $0.064 \pm 0.008$ & $0.051 \pm 0.007$ \\
\hline & Contrast & $979 \pm 132$ & $1713 \pm 236$ & $2171 \pm 301$ \\
\hline & Correlation & $(38.7 \pm 3.8) \times 10^{-5}$ & $(26.9 \pm 3.0) \times 10^{-5}$ & $(19.5 \pm 2.5) \times 10^{-5}$ \\
\hline & IDM & $0.613 \pm 0.010$ & $0.472 \pm 0.014$ & $0.389 \pm 0.018$ \\
\hline & Entropy & $4.43 \pm 0.05$ & $4.47 \pm 0.05$ & $4.50 \pm 0.05$ \\
\hline \multirow[t]{5}{*}{ DGr } & ASM & $0.179 \pm 0.014$ & $0.153 \pm 0.019$ & $0.133 \pm 0.021$ \\
\hline & Contrast & $71 \pm 29$ & $137 \pm 53$ & $202 \pm 76$ \\
\hline & Correlation & $(88.8 \pm 4.6) \times 10^{-5}$ & $(85.9 \pm 5.0) \times 10^{-5}$ & $(82.9 \pm 5.6) \times 10^{-5}$ \\
\hline & IDM & $0.911 \pm 0.034$ & $0.830 \pm 0.060$ & $0.758 \pm 0.079$ \\
\hline & Entropy & $1.99 \pm 0.10$ & $2.19 \pm 0.14$ & $2.34 \pm 0.16$ \\
\hline
\end{tabular}

Because of the weighting factor $\left[1+(i-j)^{2}\right]^{-1}$, IDM will get small contributions from far separate areas $(i \neq j)$. This makes IDNM lower for "locally" inhomogeneous images and higher for locally homogeneous images. Therefore, contrast is a measure of the appearance frequency for distant pairings while IDM is that of near neighboring pairing.

The mean IDM for DGr was $0.911 \pm 0.034$ at pixel distance $d=1$. The value for $\mathrm{CP}$ cells at a pixel distance $d=1$ was $0.595 \pm 0.005$. The difference in values was the highest between $\mathrm{DGr}$ and CP. The difference in value remained the highest for pixel distance 2 with values of $0.830 \pm 0.060$ for BGl and $0.447 \pm 0.007$ for $\mathrm{CP}$. The value of IDM decreased for all cell types from pixel distance 1 to pixel distance 3 . The difference in the values between each cell type also decreased significantly with increasing pixel length.

Entropy is defined and most frequently used in thermodynamics and it has also been used in information science

entropy $=-\sum_{i=0}^{G-1} \sum_{j=0}^{G-1} P(i, j) \times \log [P(i, j)]$.

In the former, it has relevance to the useless (unusable) energy and direction of equilibration, whereas in the latter it is used as a measure of the amount of information and randomness present in a signal. In imaging, it is used in terms of the 
Table 2 Values of texture parameters evaluated for three pixel distances $d=4,5,6$. The five cell types studied were pyramidal neurons in the BN, BGI, DGr cells, CP cells, and CGr cells.

\begin{tabular}{|c|c|c|c|c|}
\hline Cell type & Texture parameter & $d=4$ & $d=5$ & $d=6$ \\
\hline \multirow[t]{5}{*}{$\mathrm{BN}$} & ASM & $0.103 \pm 0.019$ & $0.095 \pm 0.018$ & $0.091 \pm 0.018$ \\
\hline & Contrast & $1158 \pm 97$ & $1278 \pm 99$ & $1337 \pm 101$ \\
\hline & Correlation & $(43.3 \pm 3.7) \times 10^{-5}$ & $(38.0 \pm 3.1) \times 10^{-5}$ & $(35.6 \pm 2.9) \times 10^{-5}$ \\
\hline & IDM & $0.536 \pm 0.052$ & $0.501 \pm 0.055$ & $0.482 \pm 0.056$ \\
\hline & Entropy & $3.31 \pm 0.19$ & $3.37 \pm 0.20$ & $3.40 \pm 0.20$ \\
\hline \multirow[t]{5}{*}{ BGI } & ASM & $0.185 \pm 0.082$ & $0.176 \pm 0.080$ & $0.169 \pm 0.080$ \\
\hline & Contrast & $1058 \pm 73$ & $1146 \pm 69$ & $1203 \pm 68$ \\
\hline & Correlation & $(42.8 \pm 6.0) \times 10^{-5}$ & $(36.0 \pm 5.2) \times 10^{-5}$ & $(31.8 \pm 4.4) \times 10^{-5}$ \\
\hline & IDM & $0.581 \pm 0.071$ & $0.551 \pm 0.076$ & $0.530 \pm 0.079$ \\
\hline & Entropy & $2.85 \pm 0.39$ & $2.90 \pm 0.40$ & $2.93 \pm 0.40$ \\
\hline \multirow[t]{5}{*}{$\mathrm{CP}$} & ASM & $0.042 \pm 0.003$ & $0.039 \pm 0.003$ & $0.038 \pm 0.003$ \\
\hline & Contrast & $1670 \pm 201$ & $1781 \pm 206$ & $1844 \pm 209$ \\
\hline & Correlation & $(20.5 \pm 4.9) \times 10^{-5}$ & $(15.2 \pm 4.6) \times 10^{-5}$ & $(12.3 \pm 4.4) \times 10^{-5}$ \\
\hline & IDM & $0.307 \pm 0.009$ & $0.278 \pm 0.010$ & $0.260 \pm 0.010$ \\
\hline & Entropy & $4.40 \pm 0.08$ & $4.44 \pm 0.08$ & $4.47 \pm 0.08$ \\
\hline \multirow[t]{5}{*}{ CGr } & ASM & $0.045 \pm 0.007$ & $0.042 \pm 0.006$ & $0.040 \pm 0.006$ \\
\hline & Contrast & $2449 \pm 341$ & $2588 \pm 356$ & $2662 \pm 361$ \\
\hline & Correlation & $(14.9 \pm 2.3) \times 10^{-5}$ & $(12.7 \pm 2.2) \times 10^{-5}$ & $(11.4 \pm 2.1) \times 10^{-5}$ \\
\hline & IDM & $0.338 \pm 0.019$ & $0.310 \pm 0.020$ & $0.292 \pm 0.020$ \\
\hline & Entropy & $4.43 \pm 0.052$ & $4.47 \pm 0.053$ & $4.50 \pm 0.054$ \\
\hline \multirow[t]{5}{*}{ DGr } & ASM & $0.118 \pm 0.021$ & $0.106 \pm 0.020$ & $0.097 \pm 0.019$ \\
\hline & Contrast & $269 \pm 101$ & $338 \pm 128$ & $408 \pm 154$ \\
\hline & Correlation & $(79.9 \pm 6.4) \times 10^{-5}$ & $(76.9 \pm 7.3) \times 10^{-5}$ & $(73.9 \pm 8.2) \times 10^{-5}$ \\
\hline & IDM & $0.696 \pm 0.089$ & $0.643 \pm 0.092$ & $0.598 \pm 0.092$ \\
\hline & Entropy & $2.47 \pm 0.17$ & $2.57 \pm 0.18$ & $2.66 \pm 0.18$ \\
\hline
\end{tabular}

latter sense and nonhomogeneous images have low first-order entropy, whereas homogeneous scenes have high entropy.

The values of the five texture parameters defined by the above equations and calculated for the cells shown in Fig. 6 . For the analysis, we evaluated the five images of cells at the same lateral positions with five different depth (axial) positions (layer 1 to layer 5 with a step of $0.283 \mu \mathrm{m}$ ). They are summarized in Tables 1 and 2, and Fig. 7. The values for GLCM parameters in Tables 1 and 2 are the mean values of statistical analysis of the GLCM parameter values obtained by the calculation of the five PT images of the same cells with the five different depth positions. The parameters are obtained by using Image $\mathrm{J}$ plugin "texture analysis" and the values after \pm are the standard deviations of the statistical analysis over the five PT images.
The values for $d=4-6$ have common features of monotonic increase or decrease as seen in this figure in the same way as for $d=1,2$, and 3 in Table 1 .

The values in Tables 1, 2, and Fig. 7, ASM, correlation, and IDM monotonically decrease with increase in $d$, while the value of contrast monotonically increases with increase in $d$. This is reasonable because of the following reasons. If $d$ increases in the case of correlation, the above feature is reasonable since the intensity correlation becomes less and less correlated. ASM decreases because the probability $P(i, j)$ decreases with the increase in $|i-j|$ and then the squared value of $P(i, j)$ in Eq. (1) decreases even more rapidly. IDM decreases because of the $|i-j|^{2}$ in the denominator in Eq. (4) even with $P(i, j)$ in the numerator. 

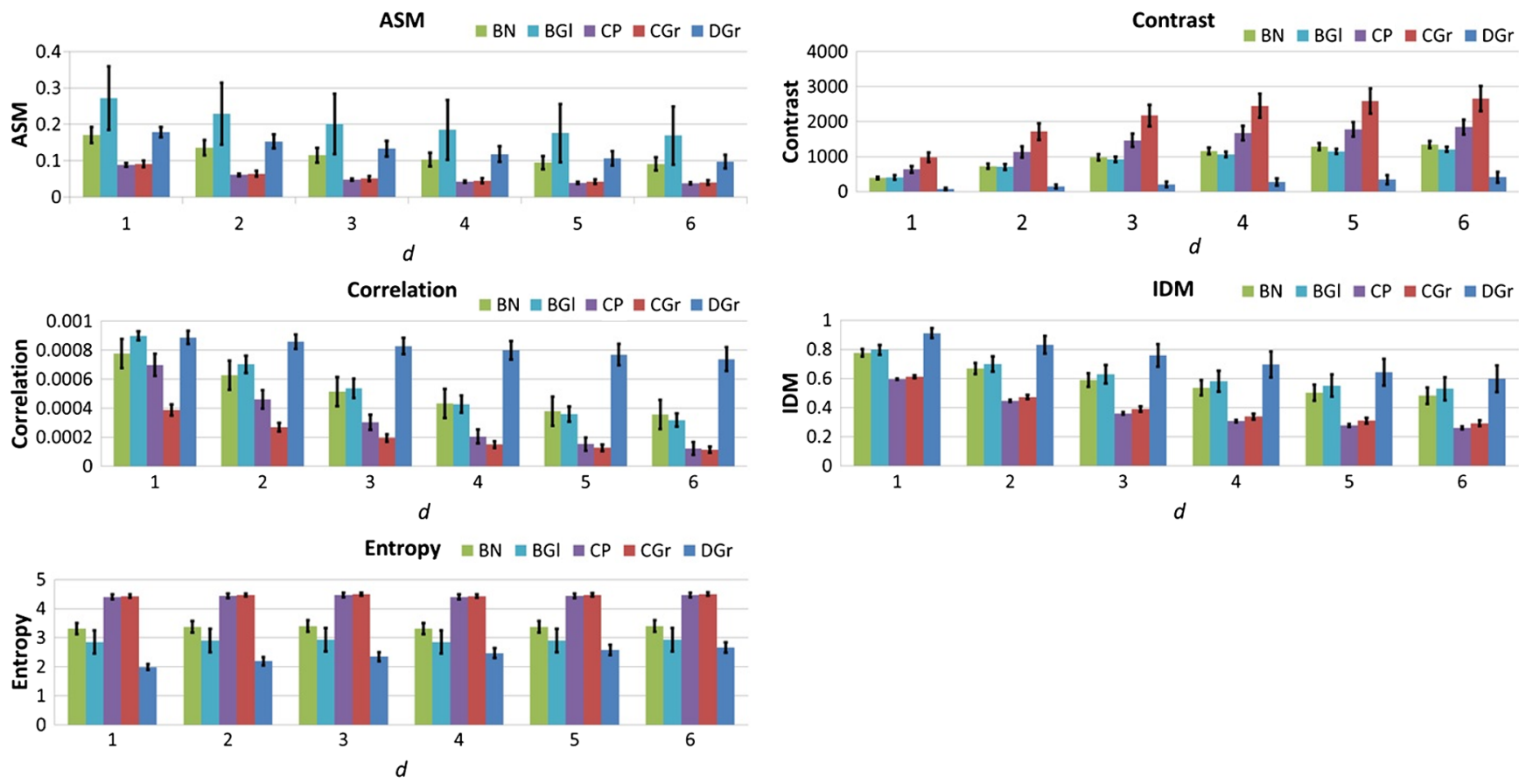

Fig. 7 Graphical representation of variations in texture parameters. Among the above graphically represented parameters, correlation, ASM, and contrast showed clear differences among the different cell types. Entropy had the smallest variation among the different cell types. The distribution of the entropy values over the five different cell types had differences smaller than 0.8 .

The physiological meaning of the parameter values are discussed in the following.

In the present experiment, we used a 488-nm pump laser which is strongly absorbed by hematoxylin, which beads positive charge and binds chiefly nucleic acids such as mRNAs and DNAs, hence it is used for examining detailed chromatin structure. Interphase nucleus consists of roughly two portions; a condensed, inactive, and densely stained portion called heterochromatin, and a loosened, actively transcribed, and faintly stained portion called euchromatin. Heterochromatin is further divided into two portions, the constitutive chromatin such as pericentromeric region and telomeric region, which always retains heterochromatin, and the facultative heterochromatin, which bears heterochromatin state upon the satisfaction of some conditions ceasing the transcription. The texture analysis of GLCM is thought to reflect the transcriptional activity of the cells. Especially in the stages such as initialization of cells occurring during iPS cell induction or early embryogenesis, where many genes are transcribed simultaneously and dynamically, the texture analysis of nuclei seems to provide interesting findings otherwise unavailable.

For the GLCM analysis, there are several important remarks. The spatial resolution is $269 \mathrm{~nm}$, imaging pixel size is $240 \mathrm{~nm}$, and distance parameter $d=240 \mathrm{~nm}$. They are thus nearly equal indicating the analysis of the image using the distance parameter is most appropriate.

The values of ASM have larger dispersion than the others. This is because the parameter includes the squared values of $P(i, j)$ and hence, it is more sensitive to the variation of $P(i, j)$ than the other parameters.

From the detailed investigation of the data depicted in Table 1 (plus additional columns for $d=4-6$ ), Table 2 and Fig. 7, we could find out that correlation, ASM, and contrast showed clear differences among the different cell types. It is also found that they have the following detailed features of the five texture parameters.

1. The texture parameters ASM and IDM have minimum values for CP and CGr among the five different cell types, and entropy has maximum values for the two cell types in all cases of distance. This means that the intracellular structure of these cell types in the cerebellum is more nonhomogeneous than the other cell types, especially for a short distance. This may mean that the distributions of heterochromatin and euchromatin are varying in a short distance indicating more complicated gene-expression in these cell types.

2. The cell types of BN and BGl have the lowest contrast and the highest correlation among the five cell types. This indicates that both neuron cells and glial cells have smaller variations over large distances than the other cell types. These cell types in BN have smaller contrast for $d=6$ (corresponding to $1440 \mathrm{~nm}$ ) than the other types for $d=3$ (corresponding to $720 \mathrm{~nm}$ ). This means that the nonhomogeneity is extended even to distances larger than $1.4 \mu \mathrm{m}$ in these cells.

3. The cell type of CGr has the highest contrast and the lowest correlation. This is in contrast to the case of DGr, BN, and BGl. It reflects that CGr has the highest nonhomogeneity among the five cell types. It means that the physiological function of CGr can be maintained even under intracellular nonhomogeneous conditions in the cerebral area. In such a way, we can classify the five cell types into groups. Granule cell in cerebellum CGr has the high contrast and low correlation indicating that the concentration of chromatin is 
Table 3 Order of parameter size among the five different cell types for $d=1-6$. The five cell types are the nerve cells (BN) and the glial cells (BGl) from BN, granule cells from dental gyrus (DGr) and Purkinje (CP) and granule cells (CGr) from cerebellum.

\begin{tabular}{|c|c|c|c|c|c|}
\hline Property & ASM & Contrast & Correlation & IDM & Entropy \\
\hline$d=1$ & $\begin{array}{l}\mathrm{BGI}>\mathrm{DGr}>\mathrm{BN}> \\
\mathrm{CGr}>\mathrm{CP}\end{array}$ & $\begin{array}{l}\mathrm{CGr}>\mathrm{CP}>\mathrm{BGl}> \\
\mathrm{BN}>\mathrm{DGr}\end{array}$ & $\begin{array}{l}\mathrm{BGl}>\mathrm{DGr}>\mathrm{BN}> \\
\mathrm{CP}>\mathrm{CGr}\end{array}$ & $\begin{array}{l}\mathrm{DGr}>\mathrm{BGl}>\mathrm{BN}> \\
\mathrm{CGr}>\mathrm{CP}\end{array}$ & $\begin{array}{l}\mathrm{CGr} \sim \mathrm{CP}>\mathrm{BN}> \\
\mathrm{BGl}>\mathrm{DGr}\end{array}$ \\
\hline$d=2$ & $\begin{array}{l}\mathrm{BGI}>\mathrm{DGr}>\mathrm{BN}> \\
\mathrm{CGr}>\mathrm{CP}\end{array}$ & $\begin{array}{l}\mathrm{CGr}>\mathrm{CP}>\mathrm{BN}> \\
\mathrm{BGl}>\mathrm{DGr}\end{array}$ & $\begin{array}{l}\mathrm{DGr}>\mathrm{BGl}>\mathrm{BN}> \\
\mathrm{CP}>\mathrm{CGr}\end{array}$ & $\begin{array}{l}\mathrm{DGr}>\mathrm{BGl}>\mathrm{BN}> \\
\mathrm{CGr}>\mathrm{CP}\end{array}$ & $\begin{array}{l}\mathrm{CGr} \sim \mathrm{CP}>\mathrm{BN}> \\
\mathrm{BGl}>\mathrm{DGr}\end{array}$ \\
\hline$d=3$ & $\begin{array}{l}\mathrm{BGI}>\mathrm{DGr}>\mathrm{BN}> \\
\mathrm{CGr}>\mathrm{CP}\end{array}$ & $\begin{array}{l}\mathrm{CGr}>\mathrm{CP}>\mathrm{BN}> \\
\mathrm{BGl}>\mathrm{DGr}\end{array}$ & $\begin{array}{l}\mathrm{DGr}>\mathrm{BGl}>\mathrm{BN}> \\
\mathrm{CP}>\mathrm{CGr}\end{array}$ & $\begin{array}{l}\mathrm{DGr}>\mathrm{BGl}>\mathrm{BN}> \\
\mathrm{CGr}>\mathrm{CP}\end{array}$ & $\begin{array}{l}\mathrm{CGr} \sim \mathrm{CP}>\mathrm{BN}> \\
\mathrm{BGl}>\mathrm{DGr}\end{array}$ \\
\hline$d=4$ & $\begin{array}{l}\mathrm{BGI}>\mathrm{DGr}>\mathrm{BN}> \\
\mathrm{CGr}>\mathrm{CP}\end{array}$ & $\begin{array}{l}\mathrm{CGr}>\mathrm{CP}>\mathrm{BN}> \\
\mathrm{BGI}>\mathrm{DGr}\end{array}$ & $\begin{array}{l}\mathrm{DGr}>\mathrm{BN}>\mathrm{BGl}> \\
\mathrm{CP}>\mathrm{CGr}\end{array}$ & $\begin{array}{l}\mathrm{DGr}>\mathrm{BGl}>\mathrm{BN}> \\
\mathrm{CGr}>\mathrm{CP}\end{array}$ & $\begin{array}{l}\mathrm{CGr} \sim \mathrm{CP}>\mathrm{BN}> \\
\mathrm{BGI}>\mathrm{DGr}\end{array}$ \\
\hline$d=5$ & $\begin{array}{l}\mathrm{BGl}>\mathrm{DGr}>\mathrm{BN}> \\
\mathrm{CGr}>\mathrm{CP}\end{array}$ & $\begin{array}{l}\mathrm{CGr}>\mathrm{CP}>\mathrm{BN}> \\
\mathrm{BGl}>\mathrm{DGr}\end{array}$ & $\begin{array}{l}\mathrm{DGr}>\mathrm{BN}>\mathrm{BGl}> \\
\mathrm{CP}>\mathrm{CGr}\end{array}$ & $\begin{array}{l}\mathrm{DGr}>\mathrm{BGl}>\mathrm{BN}> \\
\mathrm{CGr}>\mathrm{CP}\end{array}$ & $\begin{array}{l}\mathrm{CGr} \sim \mathrm{CP}>\mathrm{BN}> \\
\mathrm{BGI}>\mathrm{DGr}\end{array}$ \\
\hline$d=6$ & $\begin{array}{l}\mathrm{BGI}>\mathrm{DGr}>\mathrm{BN}> \\
\mathrm{CGr}>\mathrm{CP}\end{array}$ & $\begin{array}{l}\mathrm{CGr}>\mathrm{CP}>\mathrm{BN}> \\
\mathrm{BGl}>\mathrm{DGr}\end{array}$ & $\begin{array}{l}\mathrm{DGr}>\mathrm{BN}>\mathrm{BGl}> \\
\mathrm{CP}>\mathrm{CGr}\end{array}$ & $\begin{array}{l}\mathrm{DGr}>\mathrm{BGl}>\mathrm{BN}> \\
\mathrm{CGr}>\mathrm{CP}\end{array}$ & $\begin{array}{l}\mathrm{CGr} \sim \mathrm{CP}>\mathrm{BN}> \\
\mathrm{BGI}>\mathrm{DGr}\end{array}$ \\
\hline
\end{tabular}

nonhomogeneous. DGr, BN, and BGl have high contrast and low correlation meaning they have an homogeneity of chromatin. CP has medium values.

4. The mean entropy was highest for CGr cells, with a value of $4.43 \pm 0.05$ for pixel length (pixel distance) $d=1$. DGr had the lowest value of $1.99 \pm 0.10$ at pixel length $d=1$. The value of entropy increased for all cell types as pixel length increased. The value for DGr and CGr at pixel length 3 was $2.34 \pm$ 0.16 and $4.50 \pm 0.05$, respectively. The step increase in the entropy decreased with an increase in pixel distance. The value of entropy for cerebellar $\mathrm{CP}$ at a pixel length $d=1$ was $4.40 \pm 0.08$. The difference was the lowest between $\mathrm{CP}$ and CGr, with a magnitude of 0.04 measured at pixel distance 1 and 0.03 measured at pixel distance 3. From the value analysis, entropy can be classified into two groups; those in group 1 (G1) were in the range of 2.0 to 3.5 for $\mathrm{DGr}, \mathrm{BN}$, and $\mathrm{BGl}$ in the $\mathrm{BN}$. The others were in the range of 4.40 to 4.43 for $\mathrm{CP}$ and CGr. The entropy value of each cell type monotonically increased with $d$, but the two-grouping feature is maintained for all the $d$ values. From the graphical representation of the results shown in Fig. 7, the relative differences among cell types are increasing with the $d$ value, while the entropy was found to have the smallest variation among the different cell types in comparison with the other four parameters. From Fig. 7, the grouping into the two is also made for the other parameters. Relatively larger ASM, Correlation, IDM for G1 and relatively smaller Contrast (and Entropy as mentioned above) than those of G2.

The partition function $P(i, j)$ in Eq. (5) defining the entropy is an essential function, which can be defined for a thermodynamically large system in thermal contact with the environment. Entropy is only meaningful for systems with a large number of states to be calculated in the partition function. As the logarithm of $P(i, j)$ was calculated for entropy, the variation in entropy among the different cell types was very small and was thus unfavorable for analysis.

Tables 1 and 2 show that all five parameters (ASM, contrast, correlation, IDM, and entropy) for a short distance $(d=1)$ and a long distance $(d=6)$ are different among the cell types. For example, in case of ASM, $0.171 \pm 0.022,0.272 \pm 0.087$, $0.179 \pm 0.014,0.088 \pm 0.005$, and $0.091 \pm 0.009$ for BN, BGr, DGr, CP, and CGr, respectively, for $d=1$ They are separated from each other even though they have some overlaps of the dispersions. Similar features are also found for the other distance values $d=2-6$. Therefore, we can discuss the features of the different cell types separately using the five parameters.

Different tendencies of the orders in the sizes of the parameters that were observed in the samples of the different cell types are shown in Table 3.

The above orderings clearly show that the ASM, IDM, and correlation were all correlated with homogeneity. Contrast and entropy were anticorrelated with homogeneity. Therefore, the first three, ASM, IDM, and correlation, were correlated among each other. Contrast and entropy were anticorrelated with these three.

The "relative" standard deviations around the average of the parameters were defined by (standard deviation of the GLCM parameters)/(average of the GLCM parameters for the five different $Z$ ) Here the values are taken for angles $0 \mathrm{deg}, 45 \mathrm{deg}$, and $90 \mathrm{deg}$ (concerning the meaning of the angle, see Refs. 14 and 15 ) for $d=1-6$. Table 4 shows the values for $d=3$, as that value of $d$ was determined as the most suitable for analysis. The relative error obtained was substantially different among the five GLCM parameters for each cell type. From these results, we showed that the most suitable GLCM parameter is IDM for $\mathrm{BN}$ and $\mathrm{BGl}, \mathrm{CP}$ and $\mathrm{CGr}$ in the cerebellum, and DGr. ASM was the most suitable parameter for neurons in the basal nucleus (BGl). The same tendencies were observed for different $d$ values.

The difference in parameters and nuances in the microscopic scale analyzed by PT microscopy can be found in Table 4 . The largest distribution of ASM is for BGl and that of contrast is for DGr. Correlation has the largest distribution in the case of $\mathrm{CP}$. 
Table 4 Relative standard deviations at $d=3$ calculated using the values of averages of parameters and their standard deviation in Table 1.

\begin{tabular}{lrrrrr} 
Parameter & \multicolumn{1}{c}{ BN } & \multicolumn{1}{c}{ BGl } & \multicolumn{1}{c}{ CP } & CGr & DGr \\
\hline ASM & 17.10 & 41.40 & 6.50 & 13.66 & 15.79 \\
Contrast & 8.99 & 8.60 & 12.90 & 13.90 & 37.62 \\
Correlation & 8.91 & 12.00 & 17.70 & 12.91 & 6.76 \\
IDM & 7.84 & 10.00 & 2.20 & 4.51 & 10.42 \\
Entropy & 5.73 & 13.60 & 1.70 & 1.16 & 6.838 \\
\hline
\end{tabular}

Entropy has the smallest variation in all cell types except BGl. This is because of the logarithmic dependence of the value as discussed before and hence it is not suitable for detecting the change taking place in a cell, while IDM has the second smallest variation for all the cell types except for BGl. Therefore, IDM is the most suitable parameter for the cell state analysis for the detection of changes induced by malignation or other damages taking place in the cell except for BGl. In the case of BGl, contrast is considered to be useful as an indicator to detect cell lesions.

\section{Conclusion}

The qualifications of intracellular structure in the resolutionenhanced images of mouse brain obtained by PT imaging are analyzed to characterize the structural details of several cell types in a mouse brain. The GLCM method has been used to extract the parameters of texture features. The parameters are correlation, contrast, ASM, IDM, and entropy from the images of cells in a mouse brain. By analysis, the cell types can be classified into two groups. One group is G1 [neurons in the BN, $\mathrm{BGl}$, and DGr cells], and the other is $\mathrm{G} 2$ (CGr cells and $\mathrm{CP}$ cells). In the case of ASM, correlation, and IDM, G1 > G2. For contrast and entropy, G1<G2 for all cases of $d=1-6$ with only one exception. From the values of the parameters of entropy, IDM, and ASM, it can be concluded that the neurons and glial cells have more uniformity in BN than in the cerebellar region. From detailed analysis, it can be concluded that IDM is the most suited parameter for the cell state analysis for the detection of malignation or other damages taking place in the cell except for BGl. In the case of BGl, contrast is considered to be useful as an indicator to detect cell lesions. It is proven that it is possible to discuss the features of the different cell types separately using the five parameters because of large enough differences in the parameters among the different cell types. The texture analysis of GLCM is thought to reflect the transcriptional activity of the cells by utilizing the signal intensity which is expected to provide the distribution and correlation of the heterochromatin or euchromatin. ${ }^{30}$ This method may also be helpful for typing and prognostic prediction for cancer. ${ }^{31}$

In the present PT imaging system using a Galvano mirror, an image can be taken at the frame rate of 1 frame $/ 5 \mathrm{~s}$. Then, such correlation information can be obtained in real time with this time resolution to study the dynamics of bioenergetics in a cell through the dynamic intracellular distribution change of chromatin. This information cannot or is very difficult to be obtained by the other method. Further simple improvements will enable to provide 3-D information by simply adding a moving stage along the perpendicular direction. By using a pump at $520 \mathrm{~nm}$ close to the peak of the Eosin, we can further study the correlation between nucleus and cytoplasm in the early stage of cell division. As a future prospect, it is to be stated that even though we successfully assigned parameters which are appropriate to characterize individual cells, functional or cellular architectural meanings of them are not yet clarified. By applying our method to compare those parameters of model mice or differentiated iPS cells from them with that of wild type specimens, their meanings are expected to be elucidated, hence provide general cell biological tools to infer mechanisms of the diseases. Those are the next steps to pursue.

\section{Disclosures}

No conflicts of interest, financial or otherwise, are declared by the authors.

\section{Acknowledgments}

HT acknowledges partial support of this research by JSPS Grant-in-Aid for Scientific Research (C) (Grant No. 25330349).

\section{References}

1. M. Born and E. Wolf, Principles of Optics, Pergamon Press, New York (1965).

2. S. W. Hell and J. Wichmann, "Breaking the diffraction resolution limit by stimulated emission: stimulated emission depletion microscopy," Opt. Lett. 19, 780-782 (1994).

3. T. A. Klar et al., "Fluorescence microscopy with diffraction resolution limit broken by stimulated emission," Proc. Natl. Acad. Sci. U. S. A. 97, 8206-8210 (2000).

4. L. Meyer et al., "Dual-color STED microscopy at 30-nm focal-plane resolution," Small 4, 1095-1100 (2008).

5. P. A. Pellett et al., "Two-color STED microscopy in living cells," Biomed. Opt. Express 2(8), 2364-2371 (2011).

6. L. Schermelleh et al., "Subdiffraction multicolor imaging of the nuclear periphery with 3D structured illumination microscopy," Science 320, 1332-1336 (2008).

7. M. Bates et al., "Multicolor super-resolution imaging with photoswitchable fluorescent probes," Science 317, 1749-1753 (2007).

8. K. Uchiyama et al., "Thermal lens microscope," Jpn. J. Appl. Phys. 39, 5316-5322 (2000).

9. D. Boyer et al., "Room-temperature detection of a single molecule's absorption by photothermal contrast," Science 330, 353-356 (2010).

10. S. Bercaiaud et al., "Photothermal heterodyne imaging of individual metallic nanoparticles: theory versus experiments," Phys. Rev. B 73, 045424 (2006).

11. M. Selmke, M. Braun, and F. Cichos, "Photothermal single-particle microscopy detection of a nanolens," ACS Nano 6, 2741-2749 (2012).

12. A. A. van Dijk et al., "Absorption and scattering microscopy of single metal nanoparticles," Phys. Chem. Chem. Phys. 8, 3486-3495 (2006).

13. K. Klein et al., "Marker-free phenotyping of tumor cells by fractal analysis of reflection interference contrast microscopy images," Nano Lett. 13, 5474-5479 (2013).

14. I. Pantic et al., "Nuclear entropy, angular second moment, variance and texture correlation of thymus cortical and medullar lymphocytes: grey level co-occurrence matrix analysis," An. Acad. Bras. Ciênc. 85(3), 1063-1072 (2013).

15. A. Gebejes and R. Huertas, "Texture characterization based on greylevel co-occurrence matrix," in Proc. of the 2nd Int. Conf. of Informatics and Management Sciences ICTIC, pp. 375-378 (2013).

16. J. Miyazaki et al., "Sub-diffraction resolution pump-probe microscopy with shot-noise limited sensitivity using laser diodes," Opt. Exp. 22(8), 9024-9032 (2014).

17. J. Miyazaki et al., "Simultaneous dual-wavelength imaging of nonfluorescent tissues with 3D subdiffraction photothermal microscopy," Opt. Exp. 23(3), 3647-3656 (2015). 
18. J.-P. He et al., "Label-free imaging of melanoma with nonlinear photothermal microscopy," Opt. Lett. 40(7), 1141-1144 (2016).

19. T. Vujasinovic et al., "Gray-level co-occurrence matrix texture analysis of breast tumor images in prognosis of distant metastasis risk," Microsc. Microanal. 21(3), 646-654 (2015).

20. S. Doyle et al., "Automated grading of breast cancer histopathology using spectral clustering with textural and architectural image features," in Biomedical Imaging: From Nano to Macro, pp. 496-499 (2008).

21. I. Pantic, S. Pantic, and B. Jovanovic, "Grey level co-occurrence matrix (GLCM) texture analysis of germinal center light zone lymphocyte nuclei: physiology viewpoint with focus on apoptosis," Microsc. Microanal. 18, 470-475 (2012).

22. A. Gaiduk et al., "Room-temperature detection of a single molecule's absorption by photothermal contrast," Science 330, 353-356 (2010).

23. J. Miyazaki et al., "Optimal detection angle in sub-diffraction resolution photothermal microscopy: application for high sensitivity imaging of biological tissues," Opt. Exp. 22(16), 18833-18842 (2014).

24. J. Miyazaki et al., "Fast 3D visualization of endogenous brain signals with high-sensitivity laser scanning photothermal microscopy," Biomed. Opt. Express 7(5), 1702-1710 (2016).

25. W. Wang, Z. Wu, and $\mathrm{H}$. Zeng, "Image distortion and its correction in linear galvanometric mirrors-based laser-scanning microscopy," J. Biomed. Opt. 20(5), 056001 (2015).

26. M. Benco and R. Hudec, "Novel method for color textures features extraction based on GLCM," Radio Eng. 16(4), 64-67 (2007).

27. O. B. Sassi et al., "Improved spatial gray level dependence matrices for texture analysis," Int. J. Comput. Sci. Inf. Technol. 4(6), 209-219 (2012).

28. R. C. Gonzalez and R. E. Woods, Digital Image Processing, AddisonWesley Publishing, Boston (1993).

29. R. M. Haralick, K. Sanmugam, and I. Dinstein, "Textural features for image classification," IEEE Trans. Syst. Man Cybern. SMC-3, 610-621 (1973).
30. I. Pantic et al., "Nuclear entropy, angular second moment, variance and texture correlation of thymus cortical and medullar lymphocytes: grey level co-occurrence matrix analysis," Anais Acad. Bras. Ciênc. 85(3), 1063-1072 (2013).

31. K. Yorgesan et al., "Entropy-based texture analysis of chromatin structure in advanced prostate cancer," Cytometry 24(3), 268-276 (1996).

Takayoshi Kobayashi received $\mathrm{PhD}$ from the University of Tokyo in 1972. He was a senior researcher at the Institute of Physical and Chemical Research (RIKEN) from 1972 to 1980, and member of Technical Staff in Bell Laboratory, USA, from 1977 to 1999, a professor of physics at the University of Tokyo from 1980 to 2006, and was specially appointed and a guest professor of the University of Electro-Communications from 2006 to 2016 and 2016 to present, respectively. $\mathrm{He}$ is a distinguished chair professor of the National Chiao-Tung University, Taiwan, from 2006 to present. He has been involved in research in ultrafast phenomena and femtosecond laser development and super-resolution microscopy.

Durga Sundaram graduated from the Indian Institute of Technology in 2008 and worked in the Tokyo branch of an Indian industry during 2016 March to May.

Kazuaki Nakata received his PhD in solid state spectroscopy from Tokyo University of Science in 2010 under the tutelage of Professor E. Tokunaga. He is currently a postdoctoral research associate at the University of Electro-Communications. His research focuses predominantly on the development of the new microscopy system and superresolution imaging of biological structure.

Hiromichi Tsurui graduated from his medical course and received his PhD from the University of Tokyo in 1981 and 1989, respectively. $\mathrm{He}$ is an assistant professor at Juntendo University, School of Medicine, from 1989 to present. His specialty is biophysics, pathology, and immunology. 